\title{
Injection Moulding Reprocessing Cycles Effect Towards Mechanical Properties of Polypropylene-Nanoclay
}

\author{
M.H.Othman ${ }^{1}$, M.M.Aisha ${ }^{2}$ and S.Z.Khamis ${ }^{3}$
}

\begin{abstract}
This article is about the effects of reprocessing cycle of polypropylene-nanoclay nanocomposites (PPNC) towards its mechanical properties. The PPNC consisted of 80wt. \% Titanpro SM240 polypropylene (PP), 15wt. \% maleic anhydride grafted polypropylene (PP-G-MA)-Orevac CA100 and 5wt. \% Cloisite 15A nanoclay. This research started from performing injection molding process towards PPNC. The samples produced were measured by using Shore hardness test and Charpy impact strength test. Then, the samples were crushed back into pellets for reprocessing cycles. The reprocessing processes were repeated for four times. For comparative purpose, neat polypropylene (PP) was also underwent the same reprocessing process as PPNC. Based on the results, it was observed that when the reprocessing cycle of PPNC increased, the hardness number and impact strength of PPNC decreased. The hardness number of PPNC decreased approximately $6.56 \%$ from the first reprocessing cycle to fourth reprocessing cycle. Meanwhile, for impact strength of PPNC, the declination happened starting from second reprocessing cycle to fourth reprocessing cycle. The total declination was $14.29 \%$. Therefore, it can be concluded that, reprocessing cycle has affected the impact strength of PPNC more than the hardness of PPNC. However, when compared to neat PP, neat PP had higher hardness number and impact strength than PPNC. The findings of this research will be beneficial for recycling process of PPNC and reducing waste program.
\end{abstract}

Keywords Injection moulding, hardness, impact strength, polypropylene, nanoclay, reprocessing cycle.

\section{INTRODUCTION}

This research was about the effects of reprocessing cycles towards mechanical properties of polypropylene-nanoclay nanocomposites (PPNC). Research starts from performing injection molding process towards PPNC. Mechanical properties of samples produced are then measured in terms of impact strength and hardness. Then, the product is crushed back into pellets for reprocessing cycles. The process is repeated for four times. The outcomes of this research were the effects of reprocessing cycles towards the mechanical properties of PPNC. Reprocessing cycles of PPNC was done

M.H.Othman ${ }^{1}$ is a senior lecturer from Faculty of Mechanical and Manufacturing Engineering, UTHM. (Phone: 012-6185161; email:hilmi@uthm.edu.my).

M.M.Aisha ${ }^{2}$, was an undergraduate students from Faculty of Mechanical and Manufacturing Engineering, UTHM.

S.Z.Khamis ${ }^{3}$ is a post graduate students persuing her M.Sc in Mechanical Engineering at Faculty of Mechanical and Manufacturing Engineering, UTHM. to measure the deterioration of hardness and impact strength of PPNC after each cycles and then, to analyse the effects of hardness and impact strength of PPNC after four reprocessing cycles. Other than that, neat polypropylene (PP) also undergoes the same reprocessing process as PPNC. This is to compare the test results between these two materials.

\section{LITERATURE REVIEW}

\section{A. Polypropylene Nanocomposites}

Polypropylene was widely used in food and beverage industry as well as textile industry. This was because the material was safe to be used as food packaging and their unique properties and abilities to adapt and being mixed with various manufacturing techniques. Moreover, the product formed from polypropylene can be recycled to form another product. However, there were issues when this material was blended with various type of fillers such as nanoclay [1]. Nanocomposites are a new class of composites, that has at least one dimension of the dispersed particles in the nanometer range [2]. On of the example of nanocomposites is polypropylene-nanoclay (PPNC) nanocomposites, which have attracted a lot of attention due to their development in properties such as high modulus, increased strength and heat resistance, decreased gas permeability and flammability [3]..As the polypropylene interaction is improved, the stress is much more efficiently transferred from the polymer matrix to the inorganic filler, resulting in a higher increase in tensile properties [3]. The incorporation of clay into the polymer matrix was found to enhance thermal stability by acting as a superior insulator and mass transport barrier to the volatile products generated during decomposition [3].The weight of PPNC is lighter as compared with other conventional composites, and this makes them economical with other materials for specific [4]. Almost all PPCN formulation shows high improvement of the moduli over the investigated temperature range, which shows the plastic and elastic responses of polypropylene towards deformation are strongly influenced in the presence of nanoclay [5]

\section{B. Injection Moulding}

Injection molding is a process of forcing melted plastic into a mold cavity. This process can be performed with a host of materials, including metals. It is widely used in the manufacturing of variety of parts from the smallest to the biggest parts. However, in today's manufacturing world, this 
process is commonly used for the fabrication of plastic parts. As this process possesses a new name which is, plastic injection, a wide variety of plastic products are manufactured by this process, which vary greatly in their size, complexity, and application. There are many advantages of injection molding, which are [6] :

- High production rates

- High tolerances are repeatable

- Wide range of materials can be used

- Low labor costs

- Minimal scrap losses

- Little need to finish parts after moulding

In injection molding, the plastic is melted in the injection molding machine and then injected into the mold, where it cools and solidifies into the final part. Once the plastic has cooled, the part can be ejected. The process cycle for injection molding is very short, typically between two seconds and two minutes [7].

\section{Reprocessing Cycle of Polypropylene}

A research had been performed about the effects of reprocessing cycles on the structure and properties of isotactic polypropylene/Cloisite $15 \mathrm{~A}$ nanocomposites. In this research, the researchers have prepared $5 \mathrm{wt} . \%$ Cloisite 15A and $20 \mathrm{wt}$. $\%$ maleic anhydride-grafted-polypropylene (PP-g-MA) by direct melt intercalation in an internal mixer, and then the PPNC were subjected to 4 reprocessing cycles. The nanocomposite structure and the clay dispersion have been characterized by wide angle X-ray scattering (WAXS), transmission electron microscopy (TEM) and rheological measurements. Other characterization techniques such as Fourier transform infrared spectroscopy (FT-IR), tensile measurements, differential scanning calorimetry (DSC) and thermogravimetric analysis (TGA) have also been used to evaluate the property changes induced by reprocessing. In contrast, the complex viscosity was found to decrease for the whole samples indicating that the main effect of reprocessing was a decrease in the molecular weight. Moreover, the thermal and mechanical properties of the nanocomposites were significantly reduced after the first cycle, nevertheless they remained almost unchanged during recycling [4].

Apart from that, a research had described the effects of reprocessing on the physical and mechanical properties of composites based on radiata pine (Pinus radiata) fibre in a polypropylene (PP) matrix. Composites, containing either 40 wt $\%$ or $50 \mathrm{wt} \%$ fibre with $4 \mathrm{wt} \%$ maleated polypropylene (MAPP) as a coupling agent, were reprocessed up to eight times. Flexural tests were carried out for $40 \mathrm{wt} \%$ fibre composites and flexural strength and modulus were found to decrease with increased reprocessing. An overall 11\% reduction of tensile stress was found after reprocessing eight times compared to the virgin composites. Young's Modulus was higher for virgin composites with $50 \mathrm{wt} \%$ than those with $40 \mathrm{wt} \%$ fibre and also initially increased with reprocessing but decreased upon further reprocessing. Reprocessing was found to increase thermal stability [8].

There is also a research about the reprocessing cycle of polypropylene. This research was about extrusion moulding for 13 processing cycles of formulations containing virgin polypropylene and 1:1 mixtures of virgin and post-consumer polypropylene containing the antioxidant Recyclostab 411. The main effect of reprocessing for polypropylene was the decrease of molar mass due to degradation with chain scission, which was detected by the melt flow rate and the increase in crystallinity. Antioxidant consumption and material stability reduction with processing cycles were observed by the reduction of the thermo-oxidative stability and detected by oxidative induction time measurements. The addition of antioxidants minimized degradation and maintained the stability of the material [9].

\section{Hardness}

Hardness is the resistance of material to permanent deformation of the surface. It is the property of a metal, which gives it the ability to resist being permanently deformed when a load is applied. The hardness of a surface of the material is a direct result of interatomic forces acting on the surface of the material. Measurements of hardness are the easiest to make and are widely used for industrial design and in research. As compared to other mechanical tests, where the bulk of the material is involved in testing, all hardness tests are made on the surface or close to it. The hardness test methods are Rockwell hardness test, Brinell hardness test, Vickers hardness test, Knoop hardness test and Shore hardness test [10].

Hardness is not a fundamental property of a material, but a combined effect of compressive, elastic and plastic properties relative to the mode of penetration and shape of penetration. The main convenience of hardness is, it has a constant relationship to the tensile strength of a given material and so can be used as a practical non-destructive test. Hardness measurement can be in macro, micro \& nano-scale according to the forces applied and displacements obtained. One of the methods of hardness testing depends on the direct thrust of some form of penetrator into the metal surface and then the ploughing of the surface as a stylus is drawn across it under a controlled load [11].

\section{E. Impact Strength}

The impact strength is the ability of a material to absorb shock and impact energy without breaking [12]. Impact strength is also the ability of the material to absorb energy during plastic deformation. It is the resistance of a material to fracture under dynamic load [13].

The impact strength is calculated as the ratio of impact absorption to test specimen cross-section. It is a complex characteristic which takes into account both the toughness and strength of a material [12]. In S.I. units the impact strength is expressed in Mega Newton per $\mathrm{m} 2(\mathrm{MN} / \mathrm{m} 2)$. It is defined as the specific work required to fracture a test specimen with a stress concentration in the mid when broken by a single blow of striker in pendulum type impact testing machine [13].

Brittleness of a material is an inverse function of its impact strength. Coarse grain structures and precipitation of brittle layers at the grain boundaries do not appreciably change the mechanical properties in static tension, but substantially reduce the impact strength [14]. Impact strength is affected by the rate of loading, temperature and presence of stress raisers in the materials. It is also affected by variation in heat 
treatment, alloy content, sulphur and phosphorus content of the material [13].

Impact strength is determined by using the notch-bar impact tests on a pendulum type impact testing machine. This helps to study the effect of stress concentration and high velocity load application. The factors affecting impact strength is when the dimensions of the specimen are increased, the impact strength also increases [15].

Other than that, when the sharpness of the notch increases, the impact strength that causes failure decreases. Also, the temperature of the specimen under test gives an indication about the type of fractures like ductile, brittle or ductile to brittle transition [13]. Besides that, the angle of the notch also improves impact-strength after certain values. Lastly, the velocity of impact also affects impact strength to some extent [15]. There are two types of impact strength test which are Izod impact strength test and Charpy impact strength test.

\section{METHODOLOGY}

\section{A. Materials for Research}

Neat polypropylene and polypropylene nanoclay have been widely used in the making of plastic components. This was because of the strength, stiffness and excellent fatigue and chemical resistance. Thus, it was suitable to become the core material of this study. In this study, polypropylene-nanoclay nanocomposites (PPNC) pellets were used for the material in injection moulding. The PPNC consisted of 80wt.\% Titanpro SM240 polypropylene (PP), 15wt. \% maleic anhydride grafted polypropylene (PP-G-MA)-Orevac CA100 and 5wt. \% Cloisite $15 \mathrm{~A}$ nanoclay. The compatibiliser is the maleic anhydridegrafted-poly-propylene (PP-g-MA) and it is used to improve the compatibility of PP matrix with the organoclay. The grafting content of maleic anhydride is $1 \mathrm{wt}$. \%. The main characteristics of the compatibiliser are an average number of molecular weight $(\mathrm{Mn})$ which is $25,000 \mathrm{~g} / \mathrm{mol}$, a melt flow index of $150 \mathrm{~g} / 10 \mathrm{~min}\left(230{ }^{\circ} \mathrm{C}, 2.16 \mathrm{~kg}\right)$ according to ASTM D 1238 and a melting temperature of $167^{\circ} \mathrm{C}$ [4].

The nanofiller is organophilic clay and commercialized under the trade name Cloisite $15 \mathrm{~A}$. Cloisite $15 \mathrm{~A}$ is an additive for plastics and rubber to improve various physical properties, such as reinforcement, synergistic flame retardant and barrier. According to the manufacturer, the nanoclay was subjected to a surface treatment before mixing with hydrophobic polymers such as polypropylene. The density of Cloisite $15 \mathrm{~A}$ is $1.66 \mathrm{~g} / \mathrm{cc}$. Other than that, Cloisite $15 \mathrm{~A}$ is a modified montmorillonite (OMMT) by a quaternary ammonium salt, and dimethyl dihydrogenated tallow (2M2HT) with ca. $65 \% \mathrm{C} 18,30 \% \mathrm{C} 16,5 \% \mathrm{C} 14$ and cation-exchange capacity (C.E.C. ) of 125 meq. 100 g-1 [4].

Apart from that, the compounding of PPNC was by using a Brabender KETSE 20/40 twin-screw extruder. However, the compounding process has been done in SIRIM, Shah Alam and the pellets have already distributed to be studied. Neat polypropylene (PP) has also been distributed to be studied. This was only for comparative purpose. Neat PP underwent the same reprocessing processes and mechanical tests as PPNC

\section{B. Injection Moulding Machine and Processing}

The machine that was used for injection molding was Nissei Plastic 7 (NP7) tonne real mini horizontal plastic injection molding machine that was available in Polymer Laboratory of Faculty of Mechanical and Manufacturing Engineering in UTHM. The machine was selected as there was no other injection molding machine available in UTHM. Fig. 1 shows the Nissei Plastic 7 (NP7) tonne real mini horizontal plastic injection molding machine.

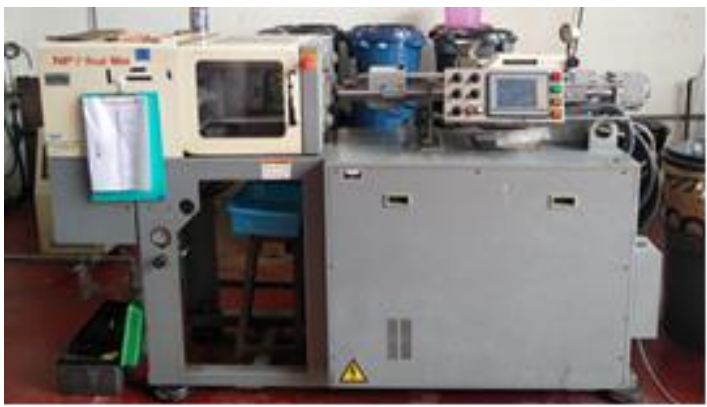

Fig. 1 : Nissei Plastic 7 (NP7) Tonne Real Mini Horizontal Plastic Injection Molding Machine

In order to perform injection molding, the injection pressure was set to $1960 \mathrm{Bar}$ and the clamp force was $49 \mathrm{kN}$. The injection speed was $250 \mathrm{~mm} / \mathrm{s}$ and the time taken for the process was less than 2 minutes. The melt temperature of PPNC was $220-280{ }^{\circ} \mathrm{C}$ and the mold temperature was $20-$ $80^{\circ} \mathrm{C}$. Also, the mould cavity was in the shape of $\mathrm{V}$-notched bar as shown in Fig. 2. The dimension of the specimens was $7.7 \mathrm{~cm} \times 0.9 \mathrm{~cm} \times 0.4 \mathrm{~cm}$.

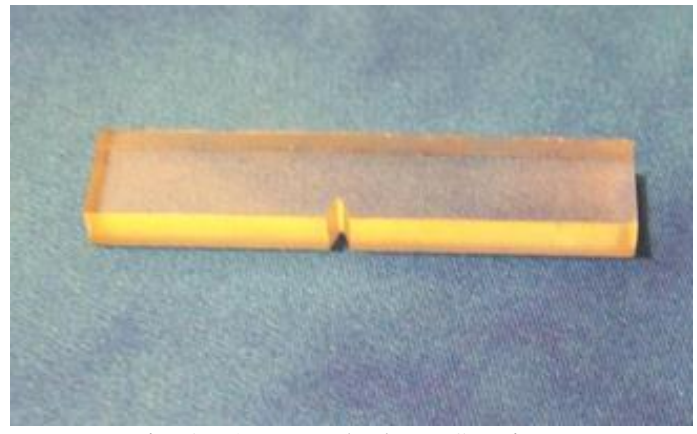

Fig. 2 : A V-notched Bar Specimen

\section{Hardness and Impact Strength Measurement}

The hardness test of PPNC after reprocessing cycles was measured using Shore Instrument Type D2240 Instron Durometer tester. The procedures of the tests followed ASTMD 2240 and the method that was used to measure hardness was Shore hardness test method. The specimen had a flat and stable surface for determining the shore hardness number. The hardness reading was obtained from the indicating pointer. Force was applied in a consistent manner to the instrument and hardness, which was the depth of the indentation, was measured. For more accurate result, reading from three different points of a specimen was measured. Other than that, the impact strength of PPNC after reprocessing cycles was measured using Amsler Otto Wolpert-Werke impact tester. The tests followed ASTM-D 256 and the method to measure 
impact resistance was according to Charpy impact test. The suitable specimen size for this test was $63.5 \mathrm{~mm} \times 12.7 \mathrm{~mm} \times$ $3.2 \mathrm{~mm}$ but the width can vary from $3 \mathrm{~mm}$ to $12.7 \mathrm{~mm}$. Specimen was clamped into the impact test fixture with the notched side facing the striking edge of the arm. Then, the arm was released and allowed to strike through the material. If breakage did not occur, a heavier hammer will be used until failure occurs. For this experiment, hammer of 4 Joule was used.

\section{Crushing of Samples Into Pellets}

After the measurement of the mechanical properties of PPNC, the samples were crushed back into pellets to be reprocessed into new samples. The machine that was used for this process was WSGI high-speed plastic granulator machine. The crushing ability of this machine is from $200 \mathrm{~kg} / \mathrm{h}-250$ $\mathrm{kg} / \mathrm{h}$. Therefore, the time taken for the machine to crush the samples was less than 5 minutes.

\section{E. Reprocessing Cycles}

After the specimens were crushed back into pellets, the pellets were being reprocessed back through injection molding process, to produce new specimens. The reprocessing cycle was repeated until the fourth reprocessing cycle. This was to obtain the trend of changes in the mechanical properties of PPNC after being reprocessed.

\section{RESULT AND DISCUSSION}

\section{A. Compounding results}

Fig. 3 shows PPNC pellets before they underwent injection molding process. The pellets were initially has rounded and spherical body. The colour of PPNC pellets was yellowish, while the colour of PP pellets was ivory white. The pellets went through injection molding process. During this process, the pellets were melted and shaped into V-notched bar. These specimens were then tested in terms of hardness and impact strength and subsequently crushed back into pellets. After this process, the shape of the pellets was flaky and different from the initial pellets shape. The pellets had to be crushed twice for a fine and smoother result. This was also made to ease the injection molding process during the subsequent reprocessing cycle. Fig. 5 shows the flaky shape of pellets after reprocessing cycle. The shape of the pellets after the first, second and third reprocessing cycle were the same. However, the mass of pellets decreased after each reprocessing cycle. For the first reprocessing cycle, $500 \mathrm{~g}$ of each PP and PPNC pellets were used for injection molding process. Then, after being tested and crushed, the mass of the pellets decreased to $300 \mathrm{~g}$. Subsequently, the mass of the pellets continued to decrease to $100 \mathrm{~g}$.

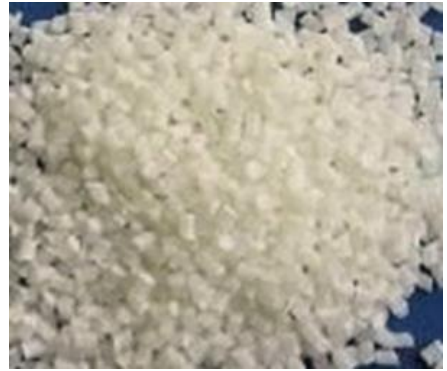

Fig. 3 : PPNC Pellets Before Injection Molding Process

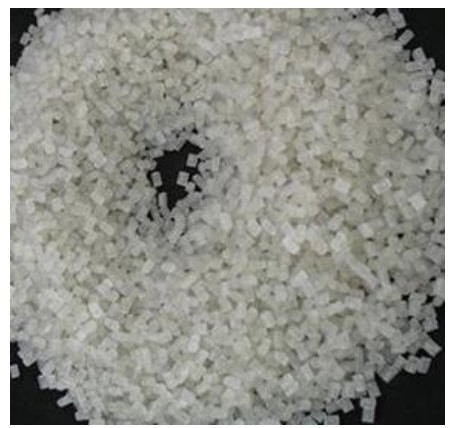

Fig. 4 : PP Pellets Before Injection Molding Process

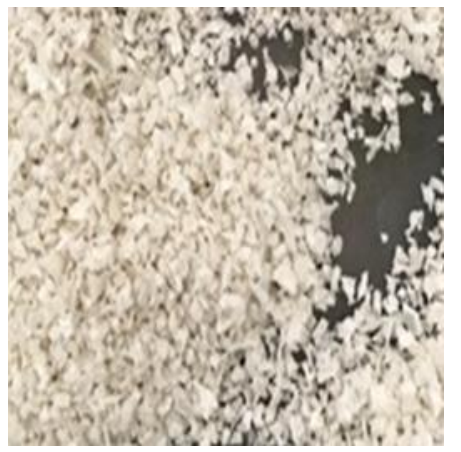

Fig. 5 : Flaky Pellets After Reprocessing Cycle

\section{B. Hardness Test}

For each specimen, data were taken at three different points. This action was performed to ensure data taken was accurate and precise. Four specimens were being tested and the average hardness of the specimens and cycles was calculated.

Table I shows the average hardness test data of PPNC and neat PP after each cycle. From the table, it can be seen that the hardness number for both material decreased after each cycle. The hardness number for PPNC decreased from 61 shore to 60 shore during second reprocessing cycle. Then, it continued to decrease from 60 shore to 58 shore and then to 57 shore during the third and fourth reprocessing cycle.

However, the hardness number of neat PP decreased from 62 shore to 61 shore during the second reprocessing cycle. Then, the hardness number went constant in the third reprocessing cycle. After that, it continued to decrease to 59 shore in the fourth reprocessing cycle. Apart from that, it can also be seen that neat PP has higher hardness number compared to PPNC even though the difference is not much. The results for the first reprocessing cycle for both material were the highest among the other reprocessing cycle results. The highest hardness number obtained was 62 shore which was the result of the first reprocessing cycle of neat PP while 
the lowest hardness number obtained was 57 shore which was the result of the fourth reprocessing cycle of PPNC.

TABLE I

Average Hardness Number Of PPNC And PP After Each Cycle

\begin{tabular}{lcccc}
\hline \hline \multirow{2}{*}{ Material } & \multicolumn{4}{c}{ Hardness Number ( Shore) } \\
\cline { 2 - 5 } & Cycle 1 & Cycle 2 & Cycle 3 & Cycle 4 \\
\hline PPNC & 61 & 60 & 58 & 57 \\
\hline PP & 62 & 61 & 61 & 59 \\
\hline \hline
\end{tabular}

\section{Impact Strength}

Using hitting power of 4 Joule for the hammer, impact strength test data has been done to nine specimens. After the test has been done, impact strength data for each cycle was obtained. Test has been done to nine specimens and the average of the reading has been calculated for all four cycles. This was to ensure data taken was accurate.

The values for average impact strength data were shown in Table II. Data show that the impact strength of PPNC increased in the second reprocessing cycle. Then, the data decreased steadily in the third reprocessing cycle and fourth reprocessing cycle. The data for first cycle was $0.19 \mathrm{~J} / \mathrm{m}^{\wedge} 2$ and it increased to $0.21 \mathrm{~J} / \mathrm{m}^{\wedge} 2$ for second cycle. It then decreased to $0.20 \mathrm{~J} / \mathrm{m}^{\wedge} 2$ in the third cycle and decreased more to $0.18 \mathrm{~J} / \mathrm{m}^{\wedge} 2$ in the fourth cycle.

TABLE II

Average Impact Strength Data of PP and PPNC After Each Cycle Impact Strength $\left(\mathrm{j} / \mathrm{m}^{2}\right)$

\begin{tabular}{lcccc}
\multicolumn{1}{c}{ Material } & \multicolumn{4}{c}{ Impact Strength $\left(\mathrm{j} / \mathrm{m}^{2}\right)$} \\
& 1 & 2 & 3 & 4 \\
\hline Polypropylene-nanoclay & & & & \\
Nanocomposites (PPNC) & 0.19 & 0.21 & 0.20 & 0.18 \\
Polypropylene (PP) & 0.40 & 0.32 & 0.29 & 0.28 \\
\hline \hline
\end{tabular}

Meanwhile, for neat PP, it can be seen that the test data decreased steadily after being reprocessed. It can be seen from the table that the test data decreased steadily from $0.40 \mathrm{~J} / \mathrm{m}^{\wedge} 2$ to $0.32 \mathrm{~J} / \mathrm{m}^{\wedge} 2$ from first reprocessing cycle to second reprocessing cycle. The value then decreased more to 0.29 $\mathrm{J} / \mathrm{m}^{\wedge} 2$ in third reprocessing cycle and $0.28 \mathrm{~J} / \mathrm{m}^{\wedge} 2$ in fourth reprocessing cycle. Based on the table, it is also clear that PP has higher impact strength than PPNC. For both material, the impact strength for the first reprocessing cycle was the highest among the other reprocessing cycle. The highest impact strength result obtained was $0.40 \mathrm{~J} / \mathrm{m}^{\wedge} 2$ which was the impact strength result for the first reprocessing cycle of neat PP. The lowest impact strength result obtained was $0.18 \mathrm{~J} / \mathrm{m}^{\wedge} 2$ which was the result for fourth reprocessing cycle of PPNC.

\section{Reprocessing Cycle Effects}

After conducting hardness and impact strength test, the result of hardness number and impact strength after each reprocessing cycle has been obtained. Data has been tabulated and graph has been plotted. Lastly, trend of data has been accessed. The data analysis showed the best material that can be reprocessed into a new product.
In Fig. 6, it shows the graph data for the materials. The blue line in the graph represents hardness test data of PP after each cycle and the red line represents hardness test data of PPNC after each cycle. It can be seen the graph line for PP starts from a point higher than graph line of PPNC. It highlights the hardness number of PP is higher than hardness number of PPNC after every reprocessing cycle.

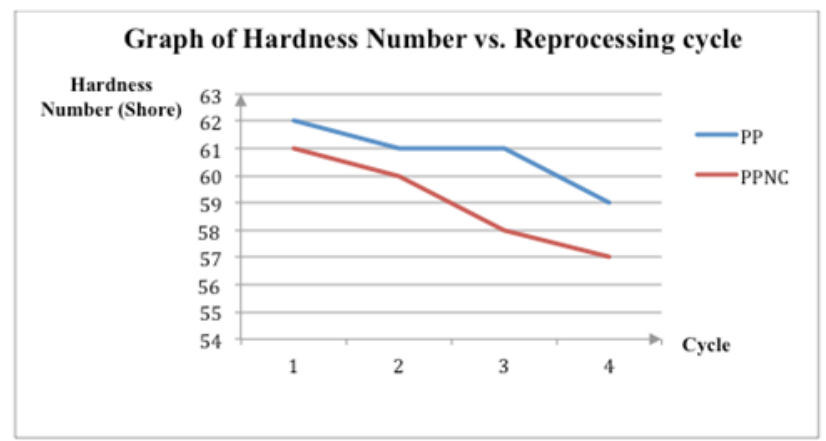

Fig. 6 : Graph of Hardness Number against Reprocessing Cycle

Graph of hardness number of PPNC decreased steadily over the reprocessing cycle. Hardness number of PPNC decreased in a total of $6.56 \%$ from the first reprocessing cycle to fourth reprocessing cycle. Other than that, the hardness number of PP decreased in a total of $4.84 \%$ from the first reprocessing cycle to fourth reprocessing cycle. However, the hardness number of PP was constant in second reprocessing cycle to third reprocessing cycle.

The hardness number remained constant at second and third reprocessing cycle because there were some errors that occurred during the testing of the specimens. During the testing of the specimens, not all of the surface of the specimens that were produced after third reprocessing cycle were flat. In order to get an accurate reading of hardness using shore hardness test, surface of specimens has to be flat and stable because force has to be exerted on the durometer to get the hardness reading that is based on the depth of indentation of the specimen. Besides that, the specimens were also directly tested after the injection molding process. Specimens cannot be tested directly after injection molding process, this is because the specimens are not cooled properly and the particles of the specimen are still very vulnerable.

Fig. 7 shows the impact strength of PPNC and PP over each cycle. The blue line in the graph represents impact strength of PP after each reprocessing cycle while the red line represents impact strength of PPNC after each reprocessing cycle. The starting point of impact strength of $\mathrm{PP}$ is higher than the starting point of impact strength of PPNC. This indicates impact strength of PP is higher than of PPNC for the first until the fourth reprocessing cycle.

The overall decrease from first reprocessing cycle to fourth reprocessing cycle in the graph of impact strength of PP was $30 \%$. Meanwhile, for the graph of PPNC, the declination happened starting from second reprocessing cycle to fourth reprocessing cycle. The total declination was $14.29 \%$. The impact strength of the first reprocessing was lower than the second reprocessing cycle by $10 \%$. The reading of first 
reprocessing cycle was supposed to be more than the reading of the second reprocessing cycle.

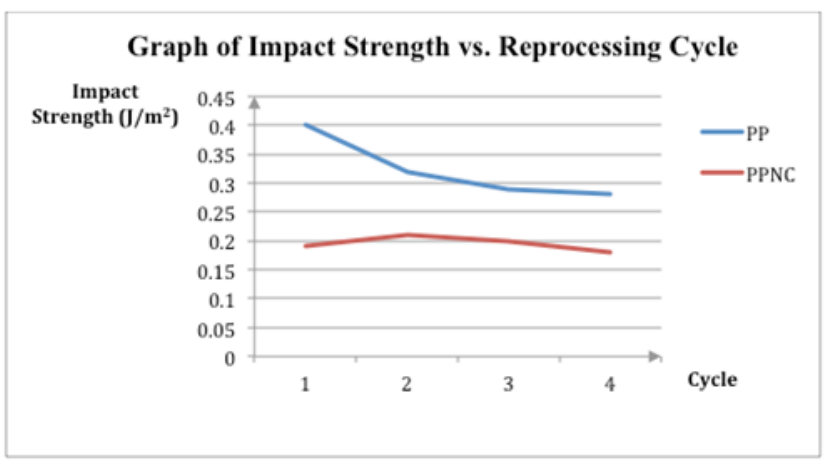

Fig. 7 : Graph of Impact Strength against Reprocessing Cycle

Based on overall findings, the trend of hardness number and impact strength of PPNC and PP after each reprocessing cycle was seen to decrease. However, for the results of impact strength, the results obtained in this finding contradict with a result that was obtained from a research [16]. From this research, it concluded that the result of impact strength after each reprocessing process increases due to the reduction in molecular weight due to the processing. This might be different because of the different mixture of material that was used in the study. The study used recycled polypropylene mix with virgin polypropylene for their material [16].

Other than that, based on the overall results, PP has higher impact strength and hardness number than PPNC after each reprocessing cycle. This finding contradicts the finding obtained from previous research. The findings generally stated that PPNC usually improved mechanical and materials properties, when it was compared to original polymer. The contradiction of findings might be because of the different preparation or compounding method of PPNC and the previous findings have not performed the reprocessing cyles[2].

\section{CONCLUSION}

In conclusion, polypropylene-nanoclay nanocomposites (PPNC) test samples has been successfully prepared through injection molding process.

Other than that, the mechanical properties of PPNC have been measured for each reprocessing cycle. The hardness and impact strength results for the first reprocessing cycle for both materials were the highest among the other reprocessing cycle results. The highest hardness number obtained was 62 shore which was the result for the first reprocessing cycle of neat PP while the lowest hardness number obtained from the experiment was 57 shore which was the result for the fourth reprocessing cycle of PPNC. The highest impact strength result obtained was $0.40 \mathrm{~J} / \mathrm{m}^{\wedge} 2$ which was the result for the first reprocessing cycle of neat PP while the lowest impact strength result was $0.18 \mathrm{~J} / \mathrm{m}^{\wedge} 2$ which was the result for fourth reprocessing cycle of PPNC. Based on the test results, PP has higher hardness number and impact strength than PPNC.

Lastly, the effects of reprocessing cycle of PPNC towards its mechanical properties have been known. It is analysed, when the reprocessing cycle of PPNC increase, the hardness number and impact strength of PPNC decrease. The hardness number of PPNC decreased in a total of $6.56 \%$ from the first reprocessing cycle to fourth reprocessing cycle. Meanwhile, for impact strength of PPNC, the declination happened starting from second reprocessing cycle to fourth reprocessing cycle. The total declination was $14.29 \%$. It can be observed that reprocessing cycle has affected the impact strength of PPNC more than it has affected the hardness of PPNC.

\section{ACKNOWLEDGMENT}

This work was supported by Ministry of Higher Education Malaysia and Universiti Tun Hussein Onn Malaysia (UTHM) through Fundamental Research Grant Scheme (FRGS Vot 0750) and Short Term Grant (STG Vot U357).

\section{REFERENCES}

[1] Stamm, B. (2008). Polypropylene (PP). Kunststoffe International, 98(10), 66-69.

[2] Mohd Hilmi Othman, S. H. and M. S. W. (2014). A Review of Polypropylene Nanoclay Nanocomposites: Preparation, Properties and Applications, 465-466, 354-358.

[3] Pavlidou, S., \& Papaspyrides, C. D. (2008). A review on polymerlayered silicate nanocomposites. Progress in Polymer Science (Oxford), 33(12), 1119-1198

[4] Touati, N., Kaci, M., Bruzaud, S., \& Grohens, Y.(2011). The effects of reprocessing cycles on the structure and properties of isotactic polypropylene/Cloisite 15A nanocomposites. Polymer Degradation and Stability, 96(6), 1064-1073.

[5] S. Sinha Ray and M. Okamoto, Polymer/layered silicate nanocomposites: a review from preparation to processing, Progress in Polymer Science, 28/ 11, (2003) 1539-1641.

[6] P., \& R. (2016). Plastic Injection Moulding Explained.Retrieved December 01, 2016, from http://www.pattersonrothwell.co.uk/services/plastic-injection-moulding- explained.html

[7] Rosato, D. V, \& Rosato, M. G. (2000). Injection molding handbook. Kluwer Academic Publisher.

[8] Beg, M. D. H., \& Pickering, K. L. (2008). Reprocessing of wood fibre reinforced polypropylene composites. Part I: Effects on physical and mechanical properties. Composites Part A: Applied Science and Manufacturing, 39(7),1091-1100.

[9] Martins, M. H., \& De Paoli, M. A. (2002). Polypropylene compounding with post-consumer material: II. Reprocessing. Polymer Degradation and Stability, 78(3), 491-495. https://doi.org/10.1016/S01413910(02)00195-7

[10] England, G. (2013). Hardness testing. Hardness Testing Method. Retrieved from http://www.gordonengland.co.uk/hardness

[11] Udomphol, T. (2007). Hardness test, 33-35.

[12] Chen, B., \& Evans, J. R. G. (2009). Impact strength of polymer-clay nanocomposites. Soft Matter,5(19),3572.

[13] Davies, G., \& Irving, P. (2014). Impact, post-impact strength and postimpact fatigue behaviour of polymer composites. In Polymer Composites in the Aerospace Industry (pp. 231-259). https://doi.org/10.1016/B978-0-85709-523-7.00009-8

[14] Chen, B., \& Evans, J. R. G. (2008). Impact and tensile energies of fracture in polymer-clay nanocomposites. Polymer, 49(23), 5113-5118.

[15] Perkins, W. G. (1999). Polymer toughness and impact resistance. Polymer Engineering and Science, 39(12), 2445-2460.

[16] Raj, M. M., Patel, H. V, Raj, L. M., \& Patel, N. K. (2013). Studies on Mechanical Properties of Recycled Polypropylene Blended With Virgin Polypropylene, 2(3), 194-203. 